\title{
THE DENTIST'S RESPONSIBILITY IN THE MANAGEMENT OF THE PATIENT WITH RHEUMATIC HEART DISEASE
}

Philip M. Northrop, M.S., D.D.S., "* and Mary C. Crowley, A.B., M.S.P.H. $\dagger$

D ROBABLY there is no type of individual who causes more apprehension or anxiety to the dentist or demands a closer cooperation with the physician than the "cardiac."

From the dental standpoint the judicious treatment of such a patient requires at least certain fundamental knowledge of cardiac disease. It is true, probably, that the dentist often makes no attempt to become informed on the problem of the cardiac patient. Due to lack of interest or ignorance, the patient may be subjected to serions complications. Further, too much reliance often is placed on the decision of the physician, who, although usually fully acquainted with the cardiac status of the patient, does not always know the complete dental portion of the problem. Certainly, the management of this important group of individuals requires that both the medical and the dental profession understand each other's problems.

From the dental standpoint, the adequately informed dentist will possess more composure and self-confidence and a greater acuity in the recognition of cardiac disease. His diagnostic skill and composure, in turn, will promote a more satisfactory and desirable relationship with the physician and assure grcater safety to his patient. In addition, he will be better prepared to meet complications when they occur.

Considerable literature concerning this subject has been written by competent medical authorities. Such articles as those published by Reichert, ${ }^{1}$ Hyman, ${ }^{2}$ Weinstein, ${ }^{3}$ Schwartz and Salman, ${ }^{4}$ as well as many others, would be well worth the careful perusal of the uninformed dentist.

The present discussion deals specifically with the dentist's responsibility in his management of only one small group of "cardiacs," patients with rheumatic heart disease. It is well known that this particular type of individual is a candidate for the usually fatal disease called subacute bacterial endocarditis. It is by no means a rare or unusual disease. Frequently it follows oral surgical procedures and is caused by the localization of bacteria on previously damaged heart valves, the organisms being liberated into the blood stream as a result of the trauma of surgery.

This information has been available for many years. No treatment, however, other than precautionary measures, has been advocated which would prevent the localization of organisms on damaged heart valves. Recently $w^{5,6}$ have shown that by proper prophylactic sulfonamide therapy the incidence of bacteremias following the extraction of teeth could be markedly

From the Department of Oral Surgery, School of Dentistry, University of Michigan.

*Associated Professor of Oral Surgery, School of Dentistry, and Oral Surgeon to University Hospital, University of Michigan.

Assistant Professor of Dentistry (Bacteriology) School of Dentistry, University of Michigan. 
decreased. Although this material has been published, the subject and the added responsibility to the dental profession are thought to be of sufficient importance to warrant republication in order to reach a still larger group of dental practitioners. This discussion will be presented with the thought in mind of giving only basic information necessary to understand the problem, omitting much of the scientific detail discussed in previous reports. ${ }^{5,6}$

Since the early studies of Richards, ${ }^{7,8}$ it has been shown many times by investigators in various fields of medicine and dentistry that, as a result of manipulation or trauma, showers of organisms are carried into the blood stream. Such a bacteremia is transient, the organisms remaining in the blood stream only for about three to five minutes, after which time they disappear.

Dentistry first was introduced to the problem as a result of the spertacular findings of Okell and Elliot, ${ }^{9}$ who showed that transient bacteremias followed the extraction of teeth. These investigators were interested primarily in the source of the Streptococcus viridans found on the vegetations of those hearts which exhibited subacute bacterial endocarditis. In their study of 138 cases, blood cultures were made before and immediately following the extraction of teeth. The surgery was performed under general anesthesia. Of the entire group, a transient bacteremia occurred in 64 per cent of the cases. The patients were placed in three classes, their classification depending on the degree of oral sepsis present. In the severely septic and pyorrheic mouths, a bacteremia was found to follow surgical operations in 75 per cent of cases. Even in 38 cases without recognizable oral sepsis, 12 patients, or 31.5 per cent, had positive blood cultures. Of the entire group, positive blood cultures were obtained preoperatively in 12 instances, or 8.6 per cent. Except on four occasions the organism isolated was Streptococcus viridans.

Elliot ${ }^{10}$ later has shown that where marked gingival disease was present, the mere rocking of the tooth was sufficient to permit the organisms to enter the blood stream.

Murray and Moosnick ${ }^{11}$ have shown that transient bacteremia follows the chewing of paraffin blocks.

In the past few years, several well-conducted investigations have been made wherein blood cultures have been taken immediately following the extraction of teeth. $5,6,12,13$ The incidence of transient bacteremias, under these conditions has been found to be about 17 per cent. The wide variation of the incidence under local and general anesthesia can be attributed to the vasoconstrictor action of the adrenalin incorporated in most local anesthetics.

\section{SIGNIFICANCE OF TRANSIENT BACTEREMIAS}

With the fact now in mind that bacteremias do occur, the problem presents itself, "What is the significance of these bacteremias?"

In the normal individual a bacteremia is probably of little or no significance, since the blood stream quickly destroys the newly introduced organisms. It might be added, however, if there is any basis for the theory of focal infection, surely the frequent introduction of bacteria into the blood stream, even in a normal individual, must be considered as a portal of entry for pathogenic organisms.

Streptococcus viridans, a common inhabitant of the gastrointestinal tract, is the most common organism in the oral cavity. It is also the organism most fre- 
quently found in the blood stream during transient bacteremia. Since subacute bacterial endocarditis is, in most instances, the result of localization of Streptococcus viridans on previously damaged heart valves, it becomes apparent that such a bacteremia may be of grave significance in the etiology of this usually fatal disease.

\section{SUBACUTE BACTERIAL ENDOCARDITIS}

Incidence.-This disease is much more frequent in its occurrence than commonly thought. In the average hospital of 200 beds, several cases are treated per annum. This incidence is much higher in areas where rheumatic fever is common. In our University Hospital, six patients with the disease have been known to be hospitalized at the same time. Frequently, the disease is not recognized by the attending physician, probably because of the lack of facilities for clinical bacteriology in many communities.

Subacute bacterial endocarditis is characterized by the formation of vegetations on the heart valves, which are nearly always due to Streptococcus viridans organism. Although it is usually due to Streptococcus viridans, Galbreath and Hull, ${ }^{14}$ in a report of 32 cases, have shown that almost half were due to other organisms. It is thought that in the mechanical function of the heart with valvular disease the valves are readily traumatized. Minute blood platelets form at this site for the colonization of the bacteria. It is understandable that conditions would necessarily have to be ideal before a subacute bacterial endocarditis could develop; that is, a blood thrombus would have to be present on the endocardium at the same time that a bacteremia existed.

This disease has been regarded by most physicians as usually fatal, although undoubtedly some mild and a few severe cases occasionally recover. Recently, treatment by ehemotherapeutic agents and heparin have been used with poor results. ${ }^{15},{ }_{16}$ The work of Loewe, Rosenblatt, Greene, and Russell, ${ }^{17}$ in which they report the successful treatment of seven consecutive cases of subacute bacterial endocarditis with the use of penicillin and heparin therapy, is most interesting, and this treatment may have some promise. At present, however, the prognosis for subacute bacterial endocarditis is extremely grave. It is very questionable, once the disease has developed, if any treatment is of value.

According to Katz and Elek, ${ }^{16}$ the cause for the persistence of the disease, and the great, if not impossible, difficulty in treating the infection of Streptococcus viridans in a valvular vegetation once it has developed, are due to three factors: (a) the relative avascularity of the valve leaflets, (b) the presence of a film platelet barrier which interferes with the entrance of white blood cells and hinders the diffusion into the lesion of the bacteriostatic and bactericidal agents present in the blood stream, and (c) the fibrin constantly being added, which offers an excellent medium for the growth of these bacteria.

Symptoms.-As a rule, the onset is indefinite. The patient's attention is not directed toward the heart but, rather, he becomes aware of fever and the feeling of not being well. Often where infection of the throat or upper respiratory passages is present, or where recent surgery has been performed, such as a tonsillectomy or the extraction of teeth, the patient feels that he simply has failed to recover. The fever is remittent in character, with daily peaks of $102^{\circ} \mathrm{F}$. being common. 
The vegetations on the valves or on the walls constantly supply the blood stream with organisms which frequently produce emboli.

As the disease continues, the symptoms become more definite and diagnosis is easier. The patient develops a progressive anemia as well as embolic phenomena. The latter consist of petechial hemorrhages on the skin or under the nails. Emboli may settle in various organs such as the kidneys, an albuminuria and transient hematuria resulting. The patient may live six months or a year although frequently death may occur in a few weeks or months. Most commonly, death is due to the complete exhaustion of the patient.

It is true that subacute bacterial endocarditis affects people who have damaged heart valves, usually as a result of rheumatic fever. Rheumatic fever, however, is not the only source, for subacute bacterial endocarditis may occur with congenital heart disease. One-third of those with this disease die from it. ${ }^{18}$ It also may complicate sclerosed valves due to arteriosclerosis, or the aortic valve in syphilis.

These latter conditions are not discussed here in any detail. It should be kept in mind, however, that individuals with congenital heart disease, arteriosclerosis, or syphilitic involvement of the endocardium should be treated in the same manner as the patient with rheumatic heart disease.

\section{RHEUMATIC HEART DISEASE}

"Rheumatic heart disease" is a condition of progressive and permanent structural changes in the heart as a result of rheumatic fever.

\section{RHEUMATIC FEVER}

In rheumatic fever, the probable cause, according to current opinion, is thought to be infectious, and apparently closely associated with the invasion of the body by hemolytic streptococei. It is characterized primarily by fever, toxicity, polyarthritis, and disseminated focal inflammatory lesions of the cardiovascular system. It might be stated here that chorea, often known as Saint Vitus's dance, is now considered as a part of a multiple symptom complex; in other words, chorea is rheumatic fever plus a further complication or involvement of the brain. In addition to the usual symptoms accompanying rheumatic fever, these patients have marked psychic changes, involuntary muscular twitchings, and a propensity to endocarditis.

Incidence.-Rheumatic fever is a common disease. Paul ${ }^{19}$ has estimated a total of 840,000 cases of rheumatic heart disease, active and inactive, in a population of $100,000,000$ people. Since nearly all cases of rheumatic fever leave some degree of endocarditis or scarring on the heart valves, it becomes evident that about 1 in every 100 patients entering the dentist's office, based on Paul's figures, is a candidate for subacute bacterial endocarditis. This incidence will be much higher in certain communities, for the disease is more prevalent in damp areas such as those near water fronts. It also occurs more frequently among the poor and undernourished. Children are particularly susceptible to this disease, since over 70 per cent of the cases occur between 4 and 30 years of age, the peak being between 7 and 10 years. ${ }^{20}$

Briefly, the disease affects the myocardium, pericardium, and, especially, the endocardium in which granulomatous formations occur. The pathologic picture 
and the amount of injury produced depend on the location, size, and stage of their development. These granulomatous formations commonly are called vegetations. Those vegetations which occur so frequently along the heart valves result in scar formation later when healing takes place. These areas are the sites where the bacteria localize and produce a subacute bacterial endocarditis.

\section{PROPHYLACTIC USES OF SULFATHIAZOLE}

It was with this information in mind that our study was made in the hope that by premedication with sulfathiazole the incidence of bacteremia following oral surgical procedures could be decreased. If a decreased incidence could be shown, then it would be reasonable to assume that prophylactic sulfonamide therapy would be indicated for those people with pre-existing valvular heart disease when surgery is contemplated. Further, such a procedure should decrease the possibility of the development of a subacute bacterial endocarditis. We were aware that the nonhemolytic streptococci, that is, Streptococcus anhemolyticus and Streptococcus viridans, had been thought to be unaffected by the bacteriostatic action of the sulfonamides. Nevertheless, it was our hope that such prophylactic treatment might still be efficacious. No attempt was made to select any specific type of case or even to limit the number of extractions to one or two teeth. All types of extractions were included in the series. The surgical procedures were carried out by students, using 2 per cent procaine with $1: 50,000$ adrenalin. The bacteriologic techniques will not be given in this article but can be obtained in the original report.

In the beginning of the study, $1 \mathrm{Gm}$. of sulfathiazole and a like amount of sodium bicarbonate were given every four hours until $6 \mathrm{Gm}$. of the drug had been taken by the patient, the last dose having been given three hours before the operation was performed. In all of these studies, blood cultures and blood levels were taken just before the local anesthetic was administered. Blood cultures were then taken immediately following surgery, and again ten minutes later.

In this group (95 cases), it was found that only 40 had a blood level over $4 \mathrm{mg}$. per 100 c.c., while 55 cases fell below that level. It is to be remembered that it is desirable, in order to have an optimum bacteriostatic action of sulfathiazole, to have a blood saturation of over $4 \mathrm{mg}$. per cent. Where the blood level was above $4 \mathrm{mg}$., the incidence of positive blood cultures was found to be 5 per cent, whereas in those cases where the blood level was below $4 \mathrm{mg}$., the incidence was 14.5 per cent, nearly approximating the usual unpremedicated case of 17 per cent.

These findings revealed the importance of raising the blood level of the drug above $4 \mathrm{mg}$. if the optimum results are to be obtained. It also showed the difficulty in raising and maintaining an adequate blood level above $4 \mathrm{mg}$. per 100 c.c. The variation of the blood level probably was due to the individual ability of the patients to absorb and secrete the drug and to the failure on the part of the ambulatory patient to cooperate by taking the drug every four hours, especially at night.

In this entire series and also in nearly 100 cases used as a control series, positive blood cultures were not obtained either before or ten minutes following surgery. It seemed evident that it was only necessary to have a maximum blood level at the time of the operative procedure. Also, with the discouraging results 
in mind when using the routine procedure, it was decided to give one massive dose of $5 \mathrm{Gm}$. each of sulfathiazole and sodium bicarbonate three hours before surgery.

Forty-four cases were studied in this group. Thirty-one of the total showed blood levels of over $4 \mathrm{mg}$. per cent, the average being $5.8 \mathrm{mg}$. An incidence of 3.2 per cent of positive cultures was found in this group. The remaining 13 patients in whom the blood level was found to be below $4 \mathrm{mg}$. per cent, with an average of $3.4 \mathrm{mg}$., showed an incidence of 0.8 per cent positive cultures, which, considering the size of the group, has little significance.

A composite of these two groups treated by sulfathiazole shows definitely that the blood level of the drug is an important factor in decreasing the incidence of bacteremia. Seventy-one cases in which the blood level was above $4 \mathrm{mg}$. per 100 c.c. showed an incidence of 4.2 per cent of positive cultures; patients whose blood level was below $4 \mathrm{mg}$. had an incidence of 14 per cent positive blood cultures.

In this same study a series of 45 cases was studied, using sulfamerazine as the bacteriostatic agent. We became interested in the possibilities of this drug since it has been thought to be less toxic than sulfathiazole and to be absorbed rapidly and excreted slowly, as recently reported by Hall and Spink. ${ }^{21}$ This action would be of great advantage since a smaller amount of the drug would be necessary to reach and maintain the desired blood level.

The results from this group of cases were discouraging. Thirty-nine cases in which the blood levels were above $4 \mathrm{mg}$. per cent, with a high average of $8.2 \mathrm{mg}$., showed six positive cultures or an incidence of 15.3 per cent. No positive cultures were found in the six cases where the blood level was below $4 \mathrm{mg}$. per cent. This group again is too small to draw many conclusions; although this study using sulfamerazine as a prophylactic agent is small, it indicates that sulfamerazine is not a good bacteriostatic agent in infections by Streptococcus viridans.

\section{COMMENTS}

From the results which have been given, it seems that the prophylactic use of sulfathiazole should be instituted in the patient having valvular heart disease when infection is present or surgical measures are contemplated.

Sulfanilamide, sulfapyridine, sulfadiazine, and other sulfonamides may be as effective as sulfathiazole or more so. At the moment, however, there is not sufficient scientific information regarding their merits for prophylactic use with patients who have valvular heart disease. It is our desire to study the effect of penicillin in the same manner as we have investigated the prophylactic use of sulfathiazole. If penicillin should be found to decrease the incidence of bacteremias, it would readily replace sulfonamide therapy, primarily because of its lack of toxicity.

It should be emphasized that sulfathiazole should not be used promiscuously and that its use is advocated for patients whose histories show a predisposition to endocarditis. At the present time, the sulfonamides are being used indisceriminately for many minor ailments, such as upper respiratory infections and sinusitis, and even before the extraction of carious teeth. Such use of these drugs is condemned. Not only is such indiscriminate use of doubtful 
therapeutic value, but it is also extremely dangerous to the patient should an idiosyncrasy develop. Furthermore, when an idiosynerasy develops, the patient is denied the advantages of the drug later when a serious illness occurs.

Medical men whom we have consulted during the past four years have accepted our findings enthusiastically as a contribution to the management of the patient with endocarditis. Not only has the physician cooperated in the medication of these patients, but he also has the opinion that such patients should be given postmedication for at least twenty-four to thirty-six hours, in order to maintain the drug in the blood stream at the proper level. Such treatment probably is the safest procedure.

Although in this study we have not encountered any drug reactions, it is suggested that a physician prescribe the drug, for, on rare occasions, a patient may show an idiosyncrasy to even a small dose or a single dose. Should such a complication arise, the physician not only can share in the responsibility, but also is better able to treat the situation.

In the management of the endocarditis case given premedication, the question arises: How much surgical work should be performed at one time? It is our opinion that, once there is adequate premedication, as much surgical work should be completed at one time as is possible. Of course, such factors as the general health of the individual, the difficulty and extent of the operation, and the degree of the infection, influence this decision. We should keep in mind constantly that a sulfonamide should be used the least possible number of times.

A word might be added here about the importance of the elimination of all questionable toci of infection in this type of patient. Subacute bacterial endocarditis may follow a severe upper respiratory infection, in fact any acute infection or injury. Therefore, the physician and dentist should eliminate any questionable foci so that an acute complication will not arise later and thus predispose this patient to subacute bacterial endocarditis.

As was stated in the previous publication, it is the responsibility of the dentist and physician to afford added safety to patients who have valvular heart disease, by the prophylactic use of the sulfonamides. The dentist should inquire routinely into the patient's past history to determine whether the patient has ever had rheumatic fever, chorea, or any heart disturbance. This inquiry should be made of patients with severe oral sepsis or when surgical procedures are contemplated. If, from this inquiry, possible or definite information suggesting valvular heart disease is obtained, the dentist should contact the patient's physician requesting his confirmation or diagnosis. If his exarmination suggests the mere possibility of valvular heart disease, sulfonamide premedication should be prescribed. The physician likewise has a responsibility in the management of the patient with congenital heart disease, or endocarditis. Once a physician has under his care a patient presenting a questionable or recognized valvular heart disease, the patient should be considered definitely in the need of prophylactic treatment when any type of surgical operation becomes necessary.

\section{SUMMARY}

The management of the "cardiac" requires certain fundamental knowledge and the need for closer cooperation between the physician and dentist. 
This discussion limits consideration specifically to the management of the patient with rheumatic heart disease.

Bacteremias have been shown frequently to follow manipulation and trauma of surgical procedures, especially following the extraction of teeth. From such a bacteremia the patient with valvular heart disease (usually resulting from rheumatic fever) may develop subacute bacterial endocarditis as a result of the localization of bacteria on previously damaged heart valves.

The prophylactic use of sulfathiazole, if given in doses sufficient to raise the blood levels above $4 \mathrm{mg}$. per 100 c.c., reduces markedly the incidence of positive blood cultures.

It is in a large part the dentist's responsibility to manage the patient with valvular heart disease by treatment with sulfonamides.

It is thought that prophylactic premedication with sulfathiazole may prevent the individual with valvular heart disease from developing a subacute bacterial endocarditis.

\section{REFERENCES}

1. Reichert, P.: The Cardiac Patient in the Dental Office, D. Outlook 26: 252, 1939.

2. Hyman, A. S.: Cardiac Emergencies in Dental Practice, New York J. Dent. 9: 60, 1939.

3. Wcinstein, J.: Dental Complications in the Cardiac Patient, D. Outlook 29: 258, 1942.

4. Schwartz, S. P., and Salman, Irving: The Effects of Oral Surgery on the Course of Patients With Diseases of the Heart, AM. J. Orthonontics and Oral Surg. (Oral Surg. Section) 28: $331,1942$.

5. Northrop, P. M., and Crowley, M. C.: Prophylactic Use of Sulfathiazole in Transient Bacteremia Following the Extraction of Teeth, J. Oral Surg. 1: 19, 1943.

6. Northrop, P. M., and Crowley, M. C.: Further Studies on the Effect of the Prophylactic Tse of Sulfathiazole and Sulfamerazine on Bacteremia Following the Extraction of Teeth, J. Oral Surg. 2: $134,1944$.

7. Richards, J. H.: Bacteriologic Studies on Chronic Arthritis and Chorea, J. Bact. 5: 511, 1920.

8. Idem: Bacteremia Following Irritation of Foci of Infection, J. A. M. A. 99: 1496, 1932.

9. Okell, C. C., and Elliot, S. D.: Bacteremia and Oral Sepsis, Lancet 2: 869, 1935.

10. Elliot, S. D.: Bacteremia in Patients With Dental Disease, Proc. Roy. Soc. Med. 32 : 747,1939 .

11. Murray, M., and Moosnick, F.: Incidence of Bacteremia on Patients With Dental Disease, J. Lab. \& Clin. Med. 26: 801, 1941.

12. Burket, L. W., and Burn, C. G.: Bacteremia Following Dental Extraction, J. Dent. Research 16: 521, 1937.

13. Hopkins, J. A.: Streptococcus Viridans: Bacteremia Following Extraction of Teeth, J. A. D. A. 26: 2002, 1939.

14. Galbreath, W. R., and Hull, E.: Sulfonamide Therapy of Bacterial Endocarditis, Ann. Int. Med. 18: 201, 1943 .

15. Lichtman, S. S.: Treatment of Subacute Bacterial Endocarditis: Current Results, Ann. Int. Med. 19: 781, 1943.

16. Katz, L. N, and Elek, S. R.: Combined Heparin and Chemotherapy in Subacute Bacterial Endocarditis, J. A. M. A. 124: 149, 1944.

17. Loewe, L., Rosenblatt, P., Greene, H., and Russell, M.: Combined Penicillin and Heparin Therapy of Subacute Bacterial Endocarditis, J. A. M. A. 124: 144, 1944.

18. Abbott, M. E.: Atlas of Congenital Cardiac Disease, New York, 1936, American Heart Association, Inc.

19. Paul, J. R.: The Epidemiology of Rheumatic Fever: A Preliminary Report With Special Reference to Environmental Factors in Rheumatic Heart Disease and Recommendations for Future Investigations, New York, 1930, American Heart Association, Inc.

20. Ketcham, W. M.: The Rheumatic Heart, J. Missouri M. A. 39: 366, 1942.

21. Hall, W. H., and Spink, W. W.: Sulfamerazine: Clinical Evaluation in 116 Cases, J. A. M. A. 123: 125, 1943. 